Prepared in cooperation with the West Virginia Department of Health and Human Resources, Bureau for Public Health, Office of Environmental Health Services, Environmental Engineering Division, and the West Virginia Department of Environmental Protection, Division of Water and Waste Management

\title{
Dissolved-Gas Concentrations in Ground Water in West Virginia, 1997-2005
}

Data Series 156 


\section{Dissolved-Gas Concentrations in Ground Water in West Virginia, 1997-2005}

By Jeremy S. White and Melvin V. Mathes

Prepared in cooperation with the West Virginia Department of Health and Human Resources, Bureau for Public Health, Office of Environmental Health Services, Environmental Engineering Division, and the West Virginia Department of Environmental Protection, Division of Water and Waste Management

Data Series 156 


\section{U.S. Department of the Interior \\ Gale A. Norton, Secretary}

\section{U.S. Geological Survey \\ P. Patrick Leahy, Acting Director}

\section{U.S. Geological Survey, Reston, Virginia: 2006}

For more information about the USGS and its products:

Telephone: 1-888-ASK-USGS

World Wide Web: http://www.usgs.gov/

Any use of trade, product, or firm names in this publication is for descriptive purposes only and does not imply endorsement by the U.S. Government.

Although this report is in the public domain, permission must be secured from the individual copyright owners to reproduce any copyrighted materials contained within this report.

Suggested citation:

White, J.S., and Mathes, M.V., 2006, Dissolved-gas concentrations in ground water in West Virginia, 1997-2005: U.S.

Geological Survey Data Series 156, 8 p. 


\section{Contents}

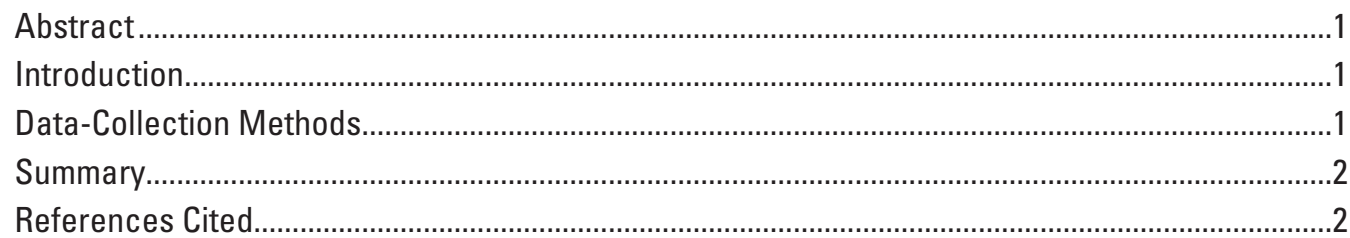

\section{Figure}

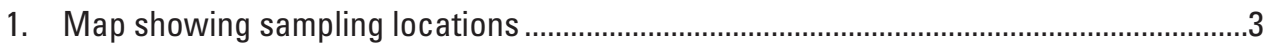

\section{Table}

1. Summary of averaged dissolved-gas compositions in water samples from wells and springs in West Virginia, 1997-2005.

\section{Conversion Factors, Datum, and Abbreviations}

\begin{tabular}{lll}
\hline \multicolumn{1}{c}{ Multiply } & \multicolumn{1}{c}{ By } & \multicolumn{1}{c}{ To obtain } \\
\hline foot $(\mathrm{ft})$ & 0.3048 & meter $(\mathrm{m})$ \\
cubic centimeter $\left(\mathrm{cm}^{3}\right)$ & 0.06102 & cubic inch $\left(\mathrm{in}^{3}\right)$ \\
liter $(\mathrm{L})$ & 0.2642 & gallon $(\mathrm{gal})$ \\
\hline
\end{tabular}

Vertical coordinate information is referenced to the National Geodetic Vertical Datum of 1929 (NGVD 29). Recharge elevation, as used in this report, refers to distance above the vertical datum.

Concentrations of gases are given in milligrams per liter (mg/L). 



\title{
Dissolved-Gas Concentrations in Ground Water in West Virginia, 1997-2005
}

\author{
By Jeremy S. White and Melvin V. Mathes
}

\section{Abstract}

Dissolved-gas samples were collected from 170 wells and 1 spring in West Virginia during 1997-2005. Gas concentrations in milligrams per liter ranged from 3.66 to 53.98 for nitrogen, 0.150 to 1.234 for argon, 0.00 to 9.11 for oxygen, 0.2 to 198.8 for carbon dioxide, and 0.00 to 68.50 for methane.

\section{Introduction}

This report presents previously unpublished dissolvedgas data for nitrogen $\left(\mathrm{N}_{2}\right)$, argon $(\mathrm{Ar})$, oxygen $\left(\mathrm{O}_{2}\right)$, carbon dioxide $\left(\mathrm{CO}_{2}\right)$, and methane $\left(\mathrm{CH}_{4}\right)$ from ground water collected in water wells and one spring in West Virginia during 1997-2005. Sample sites (fig. 1) included both public-supply and privately owned wells. Samples were taken from untreated water and were collected as part of several hydrologic studies including the Allegheny-Monongahela National Water Quality Assessment (NAWQA) (Anderson and others, 2000), the Kanawha-New River NAWQA (Paybins and others, 2000), the West Virginia Ambient Ground-Water-Quality-Monitoring Network (Kozar and Brown, 1995), a current ground-water investigation in Leetown, W. Va., and an additional 30 samples collected during this investigation to supplement deficiencies in sampling coverage. Additional water-quality parameters were collected at most sites, and sample data for 1997-2004 are published in eight Water Resources Data reports for West Virginia (Ward and others, 1998-2005). These reports contain data for major ions, nutrients, trace metals, pesticides, indicator bacteria, and VOCs (volatile organic compounds). Waterquality data collected in 2005 were not published at the time of this report.

\section{Data-Collection Methods}

Wells were sampled by connecting a flowthrough chamber and sampling lines to the raw-water tap closest to the wellhead so that field measurements and water samples could be obtained without exposing the ground water to the atmosphere. Prior to sample collection, wells were purged for several minutes until field measurements stabilized (Koterba and others, 1995). Dissolved-gas samples were collected by placing a water line into the bottom of a $160-\mathrm{cm}^{3}$ septum bottle and filling the bottle to overflowing. The septum bottle while still overflowing was then submerged into a 2-L plastic beaker that had been previously filled with ground water from the sampling site. Then the sample line was removed, and all air bubbles adhering to the sides of the bottle were allowed to escape. A rubber stopper through which a syringe needle had been inserted was then pushed tightly into the bottle, and the needle was withdrawn while the bottle was still submerged in the beaker (U.S. Geological Survey, 2005b). Multiple bottles were filled to safeguard against bottle breakage or contamination. Filled bottles were shipped inverted and chilled to the U.S. Geological Survey Chlorofluorocarbon Laboratory (Reston, Va.). Water temperature and estimated elevation of recharge to the well were submitted with the samples to aid the laboratory analysis. All samples received in good condition were analyzed using dual gas chromatography (U.S. Geological Survey, 2005a). Average values for dissolved-gas concentrations are reported for each ground-water sampling site (table 1). $\mathrm{N}_{2}$ concentrations ranged from 3.66 to $53.98 \mathrm{mg} / \mathrm{L}$, Ar from 0.150 to $1.234 \mathrm{mg} / \mathrm{L}, \mathrm{O}_{2}$ from 0.00 to $9.11 \mathrm{mg} / \mathrm{L}, \mathrm{CO}_{2}$ from 0.2 to $198.8 \mathrm{mg} / \mathrm{L}$, and $\mathrm{CH}_{4}$ from 0.00 to $68.50 \mathrm{mg} / \mathrm{L}$. 


\section{Summary}

The data presented in this report were collected during 1997-2005 as part of the Allegheny-Monongahela NAWQA, the Kanawha-New River NAWQA, the West Virginia Ambient Ground-Water-Quality-Monitoring Network, ground-water studies in Leetown, W. Va., and during this investigation. $\mathrm{N}_{2}$ concentrations ranged from 3.66 to $53.98 \mathrm{mg} / \mathrm{L}$, Ar from 0.150 to $1.234 \mathrm{mg} / \mathrm{L}, \mathrm{O}_{2}$ from 0.00 to $9.11 \mathrm{mg} / \mathrm{L}, \mathrm{CO}_{2}$ from 0.2 to $198.8 \mathrm{mg} / \mathrm{L}$, and $\mathrm{CH}_{4}$ from 0.00 to $68.50 \mathrm{mg} / \mathrm{L}$.

\section{References Cited}

Anderson, R.M., Beer, K.M., Buckwalter, T.F., Clark, M.E., McAuley, S.D., Sams, J.I., III, and Williams, D.R., 2000, Water quality in the Allegheny and Monongahela River Basins, Pennsylvania, West Virginia, New York, and Maryland, 1996-98: U.S. Geological Survey Circular 1202, $32 \mathrm{p}$.

Koterba, M.T., Wilde, F.D., and Lapham, W.W., 1995, Ground-water data-collection protocols and procedures for the National Water-Quality Assessment Program-Collection and documentation of water-quality samples and related data: U.S. Geological Survey Open-File Report 95-399, $113 \mathrm{p}$.

Kozar, M.D., and Brown, D.P., 1995, Location and site characteristics of the ambient ground-water-quality-monitoring network in West Virginia: U.S. Geological Survey OpenFile Report 95-130, 48 p.

Paybins, K.S., Messinger, Terence, Eychaner, J.H., Chambers, D.B., and Kozar, M.D., 2000, Water quality in the Kanawha-New River Basin, West Virginia, Virginia, and North Carolina, 1996-98: U.S. Geological Survey Circular 1204, 32 p.

U.S. Geological Survey, 2005a, Analytical procedures for dissolved gas, accessed November 17, 2005, at http://water.usgs.gov/lab/dissolved-gas/sampling/

U.S. Geological Survey, 2005b, Dissolved gas sampling instructions, accessed November 17, 2005, at http://water.usgs.gov/lab/dissolved-gas/sampling/

Ward, S.M., Rosier, M.T., and Crosby, G.R., 2003, Water resources data, West Virginia, water year 2002: U.S. Geological Survey Water-Data Report WV-02-1, p. 238-245.

Ward, S.M., Rosier, M.T., and Crosby, G.R., 2004, Water resources data, West Virginia, water year 2003: U.S. Geological Survey Water-Data Report WV-03-1, p. 260-273.
Ward, S.M., Rosier, M.T., and Crosby, G.R., 2005, Water resources data, West Virginia, water year 2004: U.S. Geological Survey Water-Data Report WV-04-1, p. 252-261.

Ward, S.M., Taylor, B.C., and Crosby, G.R., 1998, Water resources data, West Virginia, water year 1997: U.S. Geological Survey Water-Data Report WV-97-1, p. 342-351.

Ward, S.M., Taylor, B.C., and Crosby, G.R., 1999, Water resources data, West Virginia, water year 1998: U.S. Geological Survey Water-Data Report WV-98-1, p. 446-454.

Ward, S.M., Taylor, B.C., and Crosby, G.R., 2000, Water resources data, West Virginia, water year 1999: U.S. Geological Survey Water-Data Report WV-99-1, p. 239-250.

Ward, S.M., Taylor, B.C., and Crosby, G.R., 2001, Water resources data, West Virginia, water year 2000: U.S. Geological Survey Water-Data Report WV-00-1, p. 239-255.

Ward, S.M., Taylor, B.C., and Crosby, G.R., 2002, Water resources data, West Virginia, water year 2001: U.S. Geological Survey Water-Data Report WV-01-1, p. 240-249. 


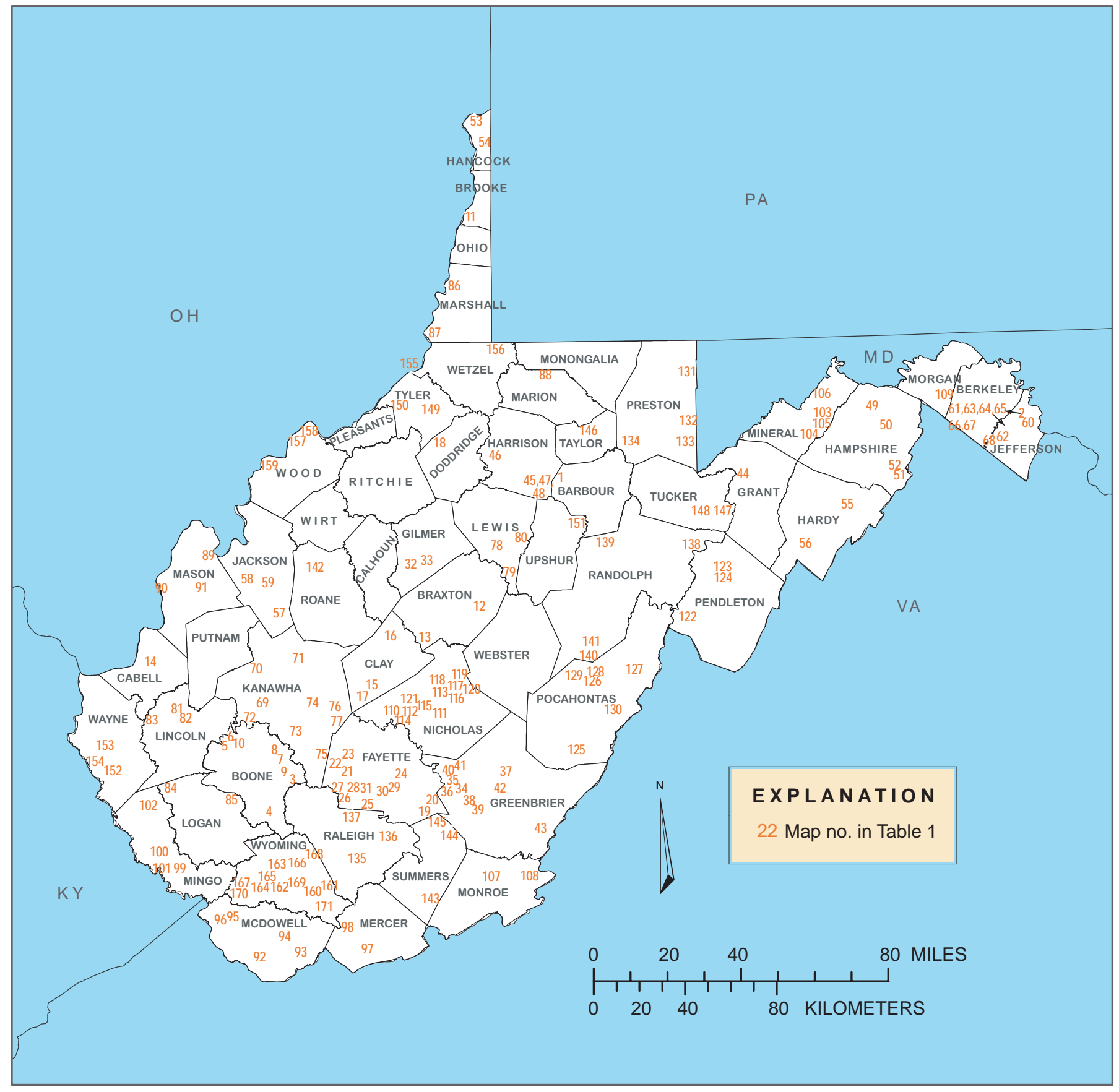

Figure 1. Sampling locations. 
Table 1. Summary of averaged dissolved-gas compositions (nitrogen, argon, oxygen, carbon dioxide, and methane) in water samples from wells and springs in West Virginia, 1997-2005.

[Map no., general reference number for map; Rech. elev., recharge elevation is land surface; ft, feet; $\mathrm{n}_{\mathrm{dg}}$, number of dissolved-gas samples averaged; $\mathrm{N}_{2}$, nitrogen; $\mathrm{Ar}$, argon; $\mathrm{O}_{2}$, oxygen; $\mathrm{CO}_{2}$, carbon dioxide; $\mathrm{CH}_{4}$, methane; $\mathrm{mg} / \mathrm{L}$, milligrams per liter; *, spring site]

\begin{tabular}{|c|c|c|c|c|c|c|c|c|c|}
\hline $\begin{array}{c}\text { Map } \\
\text { no. }\end{array}$ & Station name & Date & $\begin{array}{l}\text { Rech. elev. } \\
\text { (ft) }\end{array}$ & $n_{d g}$ & $\mathbf{N}_{2}(\mathrm{mg} / \mathrm{L})$ & $\operatorname{Ar}(\mathrm{mg} / \mathrm{L})$ & $\mathrm{O}_{2}(\mathrm{mg} / \mathrm{L})$ & $\mathrm{CO}_{2}(\mathrm{mg} / \mathrm{L})$ & $\mathrm{CH}_{4}(\mathrm{mg} / \mathrm{L})$ \\
\hline 1 & Bar-0149 & $8 / 18 / 2005$ & 1,500 & 4 & 17.57 & 0.632 & 0.01 & 13.6 & 1.5370 \\
\hline 2 & Ber-0150 & 4/14/1999 & 520 & 1 & 22.51 & 0.736 & 0.93 & 53.5 & 0.0000 \\
\hline 3 & Boo-0253 & 6/3/1997 & 2,150 & 1 & 13.00 & 0.505 & 0.02 & 16.9 & 14.2564 \\
\hline 4 & Boo-0254 & $5 / 7 / 1997$ & 2,200 & 2 & 17.24 & 0.628 & 0.10 & 4.2 & 22.7825 \\
\hline 5 & Boo-0256 & 5/4/1998 & 1,160 & 2 & 20.19 & 0.691 & 0.25 & 78.1 & 0.2789 \\
\hline 6 & Boo-0257 & $5 / 29 / 1998$ & 1,180 & 2 & 7.95 & 0.291 & 0.00 & 113.2 & 12.1966 \\
\hline 7 & Boo-0258 & $5 / 6 / 1998$ & 1,640 & 2 & 18.29 & 0.666 & 0.97 & 37.7 & 0.0000 \\
\hline 8 & Boo-0259 & $6 / 23 / 1998$ & 1,560 & 2 & 24.68 & 0.887 & 0.00 & 5.8 & 8.2377 \\
\hline 9 & Boo-0260 & $5 / 15 / 1998$ & 1,760 & 2 & 20.79 & 0.756 & 0.00 & 14.8 & 7.7542 \\
\hline 10 & Boo-0261 & $7 / 10 / 2003$ & 980 & 3 & 6.51 & 0.249 & 0.00 & 9.1 & 37.1809 \\
\hline 11 & Brk-0077 & $3 / 16 / 2005$ & 1,240 & 3 & 20.60 & 0.695 & 0.03 & 53.2 & 0.0094 \\
\hline 12 & Brx-0269 & 6/10/1997 & 1,400 & 1 & 21.55 & 0.738 & 0.15 & 23.1 & 0.0022 \\
\hline 13 & Brx-0270 & 6/9/1997 & 1,564 & 1 & 17.73 & 0.643 & 3.61 & 17.7 & 0.0000 \\
\hline 14 & Cab-0233 & 4/9/1999 & 900 & 1 & 21.56 & 0.722 & 0.00 & 6.4 & 0.6060 \\
\hline 15 & Cla-0141 & $5 / 8 / 1997$ & 1,400 & 1 & 16.27 & 0.625 & 0.07 & 23.5 & 6.7144 \\
\hline 16 & Cla-0142 & 6/4/1997 & 1,220 & 1 & 10.43 & 0.371 & 0.05 & 4.5 & 5.1290 \\
\hline 17 & Cla-0143 & $6 / 9 / 2003$ & 1,280 & 4 & 25.60 & 0.774 & 0.10 & 34.1 & 1.9190 \\
\hline 18 & Dod-0066 & $5 / 5 / 2004$ & 1,080 & 4 & 21.67 & 0.673 & 0.03 & 2.4 & 0.1035 \\
\hline 19 & Fay-0233 & $7 / 20 / 2000$ & 3,040 & 3 & 19.98 & 0.685 & 0.00 & 0.2 & 5.8524 \\
\hline 20 & Fay-0234 & 4/28/1999 & 2,880 & 1 & 49.10 & 1.234 & 5.46 & 2.6 & 0.0020 \\
\hline 21 & Fay-0242 & $7 / 11 / 2002$ & 1,920 & 4 & 21.34 & 0.694 & 0.07 & 44.0 & 0.3059 \\
\hline 22 & Fay-0267 & 6/2/1997 & 2,345 & 1 & 20.09 & 0.689 & 0.00 & 26.2 & 0.0103 \\
\hline 23 & Fay-0270 & $5 / 22 / 1998$ & 2,000 & 2 & 21.52 & 0.703 & 0.09 & 50.6 & 0.0318 \\
\hline 24 & Fay-0272 & $5 / 13 / 1998$ & 2,480 & 2 & 19.37 & 0.686 & 0.07 & 77.6 & 0.0024 \\
\hline 25 & Fay-0274 & $5 / 21 / 1998$ & 2,000 & 2 & 16.45 & 0.614 & 7.19 & 64.2 & 0.0000 \\
\hline 26 & Fay-0275 & $5 / 12 / 1998$ & 2,440 & 2 & 17.50 & 0.653 & 3.94 & 16.8 & 0.0000 \\
\hline 27 & Fay-0276 & $5 / 8 / 1998$ & 2,400 & 2 & 18.10 & 0.640 & 0.58 & 70.4 & 0.0000 \\
\hline 28 & Fay-0279 & $5 / 26 / 1998$ & 2,240 & 2 & 24.07 & 0.767 & 0.38 & 42.4 & 0.4088 \\
\hline 29 & Fay-0281 & $8 / 1 / 2000$ & 1,840 & 2 & 14.74 & 0.510 & 0.03 & 0.3 & 35.4037 \\
\hline 30 & Fay-0282 & $8 / 4 / 2000$ & 1,840 & 2 & 18.73 & 0.644 & 0.00 & 17.1 & 2.7393 \\
\hline 31 & Fay-0285 & $8 / 10 / 2000$ & 2,000 & 2 & 23.89 & 0.782 & 0.00 & 35.4 & 1.1943 \\
\hline 32 & Gil-0045 & $7 / 6 / 2000$ & 1,160 & 3 & 19.89 & 0.644 & 0.00 & 17.0 & 8.1758 \\
\hline 33 & Gil-0198 & $7 / 6 / 2000$ & 1,060 & 2 & 19.51 & 0.676 & 0.02 & 0.6 & 0.5395 \\
\hline 34 & Grb-0167 & 4/26/1999 & 3,440 & 2 & 22.51 & 0.746 & 0.30 & 24.9 & 0.3457 \\
\hline 35 & Grb-0172 & $6 / 22 / 2004$ & 3,040 & 3 & 12.95 & 0.536 & 0.20 & 81.9 & 0.9788 \\
\hline 36 & Grb-0208 & 4/27/1999 & 3,080 & 1 & 23.04 & 0.737 & 0.26 & 62.8 & 0.2998 \\
\hline 37 & Grb-0280 & $5 / 13 / 1997$ & 2,850 & 1 & 19.84 & 0.707 & 0.02 & 5.5 & 0.0007 \\
\hline 38 & Grb-0281 & $5 / 12 / 1997$ & 2,600 & 1 & 20.77 & 0.700 & 0.06 & 4.1 & 0.0004 \\
\hline
\end{tabular}


Table 1. Summary of averaged dissolved-gas compositions (nitrogen, argon, oxygen, carbon dioxide, and methane) in water samples from wells and springs in West Virginia, 1997-2005.-Continued

[Map no., general reference number for map; Rech. elev., recharge elevation is land surface; $\mathrm{ft}$, feet; $\mathrm{n}_{\mathrm{dg}}$, number of dissolved-gas samples averaged; $\mathrm{N}_{2}$, nitrogen; Ar, argon; $\mathrm{O}_{2}$, oxygen; $\mathrm{CO}_{2}$, carbon dioxide; $\mathrm{CH}_{4}$, methane; $\mathrm{mg} / \mathrm{L}$, milligrams per liter; *, spring site]

\begin{tabular}{|c|c|c|c|c|c|c|c|c|c|}
\hline $\begin{array}{l}\text { Map } \\
\text { no. }\end{array}$ & Station name & Date & $\begin{array}{c}\text { Rech. elev. } \\
\text { (ft) }\end{array}$ & $\mathbf{n}_{\mathrm{dg}}$ & $\mathrm{N}_{2}(\mathrm{mg} / \mathrm{L})$ & $\operatorname{Ar}(\mathrm{mg} / \mathrm{L})$ & $0_{2}(\mathrm{mg} / \mathrm{L})$ & $\mathrm{CO}_{2}(\mathrm{mg} / \mathrm{L})$ & $\mathrm{CH}_{4}(\mathrm{mg} / \mathrm{L})$ \\
\hline 39 & Grb-0283 & $5 / 19 / 1997$ & 2,680 & 1 & 16.70 & 0.621 & 1.06 & 7.5 & 0.0000 \\
\hline 40 & Grb-0284 & 6/16/1998 & 3,320 & 1 & 26.48 & 0.818 & 1.13 & 46.5 & 0.0017 \\
\hline 41 & Grb-0285 & 4/27/1999 & 3,500 & 1 & 21.40 & 0.736 & 0.20 & 11.6 & 0.5438 \\
\hline 42 & Grb-0287 & $7 / 13 / 2000$ & 2,440 & 2 & 21.76 & 0.696 & 0.08 & 13.6 & 0.1831 \\
\hline 43 & Grb-0291 & $3 / 24 / 2005$ & 3,280 & 3 & 19.46 & 0.688 & 1.77 & 55.0 & 0.0035 \\
\hline 44 & Grt-0106 & $6 / 25 / 2003$ & 3,600 & 4 & 20.64 & 0.674 & 0.05 & 16.8 & 0.2689 \\
\hline 45 & Har-0170 & $4 / 11 / 2005$ & 1,600 & 3 & 20.61 & 0.703 & 0.00 & 12.8 & 2.3103 \\
\hline 46 & Har-0173 & $8 / 16 / 2005$ & 1,300 & 4 & 18.17 & 0.638 & 0.00 & 3.9 & 14.9208 \\
\hline 47 & Har-0175 & $8 / 17 / 2005$ & 1,400 & 4 & 20.99 & 0.715 & 0.06 & 6.0 & 0.0131 \\
\hline 48 & Har-0176 & $8 / 17 / 2005$ & 1,460 & 4 & 22.27 & 0.763 & 0.11 & 0.3 & 7.0059 \\
\hline 49 & Hmp-0379 & $6 / 11 / 2001$ & 1,240 & 4 & 19.89 & 0.744 & 0.58 & 23.8 & 0.0000 \\
\hline 50 & Hmp-0380 & $6 / 12 / 2001$ & 1,220 & 4 & 22.62 & 0.751 & 0.07 & 13.1 & 0.0000 \\
\hline 51 & Hmp-0382 & $6 / 13 / 2001$ & 1,220 & 2 & 30.66 & 0.942 & 0.09 & 54.2 & 0.0000 \\
\hline 52 & Hmp-0383 & $6 / 13 / 2001$ & 1,700 & 4 & 20.33 & 0.727 & 0.07 & 16.4 & 0.0197 \\
\hline 53 & Hnc-0042 & $7 / 23 / 2002$ & 1,200 & 4 & 21.06 & 0.718 & 0.27 & 9.3 & 0.0022 \\
\hline 54 & Hnc-0046 & $3 / 16 / 2005$ & 1,260 & 3 & 22.50 & 0.770 & 0.07 & 31.5 & 0.9123 \\
\hline 55 & Hrd-0300 & $6 / 14 / 2001$ & 1,780 & 4 & 23.11 & 0.743 & 0.08 & 33.4 & 0.0000 \\
\hline 56 & Hrd-0303 & $6 / 27 / 2005$ & 2,250 & 4 & 22.66 & 0.749 & 0.07 & 10.3 & 0.0019 \\
\hline 57 & Jac-0166 & $5 / 27 / 1997$ & 960 & 1 & 20.99 & 0.734 & 0.02 & 15.1 & 0.0000 \\
\hline 58 & Jac-0167 & $5 / 3 / 2004$ & 820 & 4 & 25.63 & 0.813 & 0.10 & 1.2 & 0.0405 \\
\hline 59 & Jac-0168 & $6 / 14 / 2004$ & 770 & 3 & 21.76 & 0.745 & 0.58 & 0.9 & 0.0000 \\
\hline 60 & Jef-0312 & 4/15/1999 & 480 & 1 & 23.06 & 0.797 & 8.08 & 15.9 & 0.0004 \\
\hline $61 *$ & Jef-0327S & $9 / 14 / 2004$ & 500 & 4 & 20.67 & 0.701 & 0.37 & 56.3 & 0.0000 \\
\hline 62 & Jef-0579 & $7 / 15 / 2002$ & 600 & 4 & 18.36 & 0.650 & 5.29 & 75.5 & 0.0000 \\
\hline 63 & Jef-0581 & $7 / 16 / 2002$ & 540 & 3 & 18.34 & 0.642 & 3.46 & 85.8 & 0.0000 \\
\hline 64 & Jef-0585 & $7 / 21 / 2004$ & 540 & 4 & 21.17 & 0.691 & 0.06 & 39.5 & 0.0000 \\
\hline 65 & Jef-0590 & $9 / 16 / 2004$ & 500 & 4 & 20.30 & 0.690 & 0.55 & 67.1 & 0.0000 \\
\hline 66 & Jef-0592 & $7 / 27 / 2004$ & 540 & 4 & 20.79 & 0.676 & 1.26 & 56.7 & 0.0000 \\
\hline 67 & Jef-0603 & $9 / 16 / 2004$ & 510 & 4 & 25.74 & 0.797 & 0.06 & 29.3 & 3.3090 \\
\hline 68 & Jef-0641 & $5 / 20 / 2004$ & 540 & 4 & 24.79 & 0.777 & 0.06 & 42.3 & 0.0000 \\
\hline 69 & Kan-0927 & $5 / 28 / 1997$ & 1,000 & 1 & 20.62 & 0.722 & 0.04 & 48.0 & 0.0000 \\
\hline 70 & Kan-0928 & $5 / 29 / 1997$ & 700 & 1 & 20.47 & 0.707 & 0.06 & 2.4 & 1.0421 \\
\hline 71 & Kan-0932 & 5/5/1997 & 1,120 & 2 & 22.14 & 0.729 & 9.11 & 51.2 & 0.0002 \\
\hline 72 & Kan-0934 & 5/6/1997 & 870 & 2 & 16.15 & 0.622 & 0.03 & 30.0 & 4.9371 \\
\hline 73 & Kan-0935 & $5 / 5 / 1998$ & 1,440 & 1 & 20.37 & 0.699 & 0.08 & 24.5 & 0.0042 \\
\hline 74 & Kan-0938 & $6 / 22 / 1998$ & 1,580 & 2 & 19.45 & 0.677 & 0.29 & 16.7 & 0.3759 \\
\hline 75 & Kan-0939 & $6 / 26 / 2002$ & 1,920 & 4 & 15.95 & 0.545 & 0.04 & 3.7 & 37.3493 \\
\hline 76 & Kan-0940 & $7 / 10 / 2002$ & 1,580 & 4 & 22.85 & 0.723 & 0.09 & 113.0 & 0.3097 \\
\hline
\end{tabular}


Table 1. Summary of averaged dissolved-gas compositions (nitrogen, argon, oxygen, carbon dioxide, and methane) in water samples from wells and springs in West Virginia, 1997-2005.-Continued

[Map no., general reference number for map; Rech. elev., recharge elevation is land surface; $\mathrm{ft}$, feet; $\mathrm{n}_{\mathrm{dg}}$, number of dissolved-gas samples averaged; $\mathrm{N}_{2}$, nitrogen; Ar, argon; $\mathrm{O}_{2}$, oxygen; $\mathrm{CO}_{2}$, carbon dioxide; $\mathrm{CH}_{4}$, methane; $\mathrm{mg} / \mathrm{L}$, milligrams per liter; ${ }^{*}$, spring site]

\begin{tabular}{|c|c|c|c|c|c|c|c|c|c|}
\hline $\begin{array}{c}\text { Map } \\
\text { no. }\end{array}$ & Station name & Date & $\begin{array}{c}\text { Rech. elev. } \\
\text { (ft) }\end{array}$ & $\mathbf{n}_{\mathrm{dg}}$ & $\mathrm{N}_{2}(\mathrm{mg} / \mathrm{L})$ & $\operatorname{Ar}(\mathrm{mg} / \mathrm{L})$ & $\mathrm{O}_{2}(\mathrm{mg} / \mathrm{L})$ & $\mathrm{CO}_{2}(\mathrm{mg} / \mathrm{L})$ & $\mathrm{CH}_{4}(\mathrm{mg} / \mathrm{L})$ \\
\hline 77 & Kan-0941 & $7 / 11 / 2002$ & 1,520 & 4 & 22.83 & 0.750 & 0.07 & 44.0 & 1.3788 \\
\hline 78 & Lew-0215 & $4 / 11 / 2005$ & 1,300 & 3 & 21.40 & 0.723 & 0.00 & 11.4 & 0.2589 \\
\hline 79 & Lew-0217 & $3 / 10 / 2005$ & 1,360 & 1 & 21.05 & 0.775 & 0.00 & 0.5 & 21.1730 \\
\hline 80 & Lew-0221 & $8 / 15 / 2005$ & 1,280 & 4 & 10.39 & 0.395 & 0.00 & 15.4 & 22.6376 \\
\hline 81 & Lin-0179 & 4/6/1999 & 660 & 2 & 19.47 & 0.637 & 1.66 & 34.7 & 9.9004 \\
\hline 82 & Lin-0180 & 4/5/1999 & 1,000 & 1 & 21.38 & 0.730 & 1.24 & 11.5 & 6.0110 \\
\hline 83 & Lin-0185 & $6 / 3 / 2004$ & 950 & 3 & 21.22 & 0.735 & 0.13 & 45.4 & 0.0968 \\
\hline 84 & Log-0198 & 4/8/1999 & 1,300 & 1 & 17.02 & 0.558 & 1.89 & 13.6 & 30.1577 \\
\hline 85 & Log-0220 & $7 / 10 / 2003$ & 1,400 & 4 & 7.75 & 0.317 & 0.00 & 14.7 & 32.4456 \\
\hline 86 & Mal-0407 & $3 / 16 / 2005$ & 1,160 & 3 & 20.32 & 0.713 & 4.13 & 29.8 & 0.0000 \\
\hline 87 & Mal-0409 & $3 / 17 / 2005$ & 1,220 & 2 & 20.22 & 0.670 & 0.05 & 20.1 & 0.0025 \\
\hline 88 & Mar-0296 & $6 / 27 / 2000$ & 1,440 & 2 & 20.40 & 0.687 & 0.08 & 42.7 & 0.0000 \\
\hline 89 & Mas-0917 & $5 / 11 / 1999$ & 620 & 1 & 19.31 & 0.676 & 0.06 & 28.3 & 0.0000 \\
\hline 90 & Mas-0930 & $4 / 1 / 2005$ & 780 & 3 & 20.98 & 0.686 & 0.70 & 73.9 & 0.0004 \\
\hline 91 & Mas-0960 & $4 / 1 / 2005$ & 880 & 3 & 23.11 & 0.773 & 0.00 & 60.3 & 0.1248 \\
\hline 92 & Mcd-0042 & $5 / 13 / 1999$ & 2,300 & 2 & 5.84 & 0.227 & 0.43 & 18.4 & 55.6290 \\
\hline 93 & Mcd-0048 & $6 / 17 / 2004$ & 2,280 & 4 & 20.44 & 0.681 & 0.28 & 4.4 & 0.8544 \\
\hline 94 & Mcd-0109 & $5 / 19 / 1999$ & 2,240 & 2 & 20.36 & 0.680 & 0.31 & 137.4 & 0.7071 \\
\hline 95 & Mcd-0149 & $5 / 13 / 1999$ & 1,760 & 2 & 3.66 & 0.150 & 0.43 & 14.6 & 68.5010 \\
\hline 96 & Mcd-0202 & $5 / 12 / 2004$ & 2,080 & 3 & 10.65 & 0.440 & 1.17 & 12.3 & 24.2676 \\
\hline 97 & Mer-0162 & $8 / 2 / 2000$ & 2,600 & 2 & 27.72 & 0.868 & 0.03 & 19.7 & 0.3902 \\
\hline 98 & Mer-0163 & $7 / 18 / 2000$ & 2,680 & 2 & 18.27 & 0.635 & 0.00 & 41.3 & 2.0132 \\
\hline 99 & Mig-0140 & $5 / 18 / 1999$ & 1,800 & 1 & 16.80 & 0.620 & 6.14 & 33.7 & 0.0006 \\
\hline 100 & Mig-0141 & $5 / 17 / 1999$ & 1,800 & 2 & 11.53 & 0.416 & 0.32 & 8.6 & 47.2949 \\
\hline 101 & Mig-0142 & $5 / 13 / 2004$ & 1,520 & 4 & 12.13 & 0.535 & 0.60 & 23.7 & 16.8967 \\
\hline 102 & Mig-0143 & $6 / 23 / 2005$ & 1,450 & 4 & 12.41 & 0.515 & 0.00 & 6.5 & 34.5185 \\
\hline 103 & Min-0168 & $6 / 24 / 2003$ & 840 & 4 & 32.13 & 0.940 & 1.34 & 52.0 & 0.0142 \\
\hline 104 & Min-0169 & $6 / 24 / 2003$ & 960 & 4 & 28.58 & 0.903 & 0.14 & 32.9 & 0.0949 \\
\hline 105 & Min-0170 & $6 / 25 / 2003$ & 860 & 4 & 25.48 & 0.789 & 0.06 & 50.0 & 0.0073 \\
\hline 106 & Min-0171 & $6 / 26 / 2003$ & 1,960 & 1 & 20.95 & 0.712 & 4.83 & 19.2 & 0.0000 \\
\hline 107 & Mnr-0148 & $7 / 19 / 2000$ & 2,240 & 2 & 19.02 & 0.653 & 3.18 & 26.0 & 0.0014 \\
\hline 108 & Mnr-0156 & $3 / 24 / 2005$ & 3,200 & 3 & 22.57 & 0.741 & 0.04 & 2.7 & 0.0063 \\
\hline 109 & Mrg-0086 & $5 / 19 / 2004$ & 1,020 & 4 & 28.01 & 0.872 & 0.10 & 71.1 & 0.0179 \\
\hline 110 & Nic-0207 & 6/5/1997 & 1,600 & 1 & 21.38 & 0.745 & 0.00 & 37.1 & 0.4314 \\
\hline 111 & Nic-0208 & 6/16/1997 & 2,200 & 1 & 20.95 & 0.739 & 0.03 & 32.3 & 1.9742 \\
\hline 112 & Nic-0209 & 6/8/1998 & 1,880 & 2 & 21.16 & 0.736 & 0.12 & 26.7 & 0.0717 \\
\hline 113 & Nic-0211 & 6/10/1998 & 2,480 & 2 & 16.08 & 0.620 & 0.00 & 37.5 & 5.4159 \\
\hline 114 & Nic-0212 & $5 / 19 / 1998$ & 1,680 & 2 & 19.17 & 0.688 & 1.84 & 52.3 & 0.0000 \\
\hline
\end{tabular}


Table 1. Summary of averaged dissolved-gas compositions (nitrogen, argon, oxygen, carbon dioxide, and methane) in water samples from wells and springs in West Virginia, 1997-2005.-Continued

[Map no., general reference number for map; Rech. elev., recharge elevation is land surface; $\mathrm{ft}$, feet; $\mathrm{n}_{\mathrm{dg}}$, number of dissolved-gas samples averaged; $\mathrm{N}_{2}$, nitrogen; $\mathrm{Ar}$, argon; $\mathrm{O}_{2}$, oxygen; $\mathrm{CO}_{2}$, carbon dioxide; $\mathrm{CH}_{4}$, methane; $\mathrm{mg} / \mathrm{L}$, milligrams per liter; *, spring site]

\begin{tabular}{|c|c|c|c|c|c|c|c|c|c|}
\hline $\begin{array}{c}\text { Map } \\
\text { no. }\end{array}$ & Station name & Date & $\begin{array}{l}\text { Rech. elev. } \\
\text { (ft) }\end{array}$ & $\mathbf{n}_{\mathrm{dg}}$ & $\mathrm{N}_{2}(\mathrm{mg} / \mathrm{L})$ & $\operatorname{Ar}(\mathrm{mg} / \mathrm{L})$ & $\mathrm{O}_{2}(\mathrm{mg} / \mathrm{L})$ & $\mathrm{CO}_{2}(\mathrm{mg} / \mathrm{L})$ & $\mathrm{CH}_{4}(\mathrm{mg} / \mathrm{L})$ \\
\hline 115 & Nic-0213 & $5 / 28 / 1998$ & 2,200 & 2 & 25.13 & 0.779 & 0.21 & 78.0 & 0.1959 \\
\hline 116 & Nic-0214 & $6 / 3 / 1998$ & 2,440 & 2 & 20.04 & 0.661 & 0.30 & 83.1 & 0.3831 \\
\hline 117 & Nic-0215 & $6 / 2 / 1998$ & 2,440 & 2 & 23.88 & 0.819 & 0.00 & 11.6 & 6.2457 \\
\hline 118 & Nic-0217 & $6 / 12 / 1998$ & 2,520 & 1 & 23.41 & 0.752 & 0.60 & 59.8 & 0.6122 \\
\hline 119 & Nic-0218 & 6/17/1998 & 2,560 & 1 & 18.13 & 0.630 & 3.12 & 198.8 & 0.0000 \\
\hline 120 & Nic-0219 & $6 / 4 / 1998$ & 2,800 & 2 & 20.77 & 0.718 & 0.23 & 59.0 & 0.2528 \\
\hline 121 & Nic-0220 & $4 / 28 / 1999$ & 2,120 & 1 & 20.02 & 0.705 & 1.40 & 6.8 & 7.6906 \\
\hline 122 & Pen-0145 & $8 / 7 / 2002$ & 4,600 & 3 & 21.58 & 0.722 & 0.07 & 0.9 & 0.9386 \\
\hline 123 & Pen-0163 & $7 / 18 / 2002$ & 2,840 & 4 & 18.36 & 0.656 & 1.59 & 23.2 & 0.0000 \\
\hline 124 & Pen-0164 & $8 / 6 / 2002$ & 2,840 & 2 & 43.78 & 1.136 & 4.07 & 8.1 & 0.0000 \\
\hline 125 & Poc- 0234 & $3 / 23 / 2005$ & 3,000 & 3 & 22.51 & 0.729 & 0.00 & 7.2 & 1.8904 \\
\hline 126 & Poc- 0257 & $5 / 20 / 1997$ & 3,900 & 1 & 19.87 & 0.706 & 0.11 & 4.8 & 0.0000 \\
\hline 127 & Poc- 0262 & $7 / 11 / 2000$ & 3,360 & 2 & 21.10 & 0.717 & 0.21 & 8.2 & 0.0000 \\
\hline 128 & Poc- 0263 & $7 / 8 / 2003$ & 4,700 & 4 & 21.24 & 0.702 & 0.06 & 3.8 & 0.0000 \\
\hline 129 & Poc- 0265 & $7 / 9 / 2003$ & 3,600 & 3 & 17.12 & 0.623 & 2.78 & 1.4 & 0.0000 \\
\hline 130 & Poc-0266 & $3 / 23 / 2005$ & 3,680 & 3 & 25.02 & 0.819 & 0.00 & 47.4 & 0.3520 \\
\hline 131 & Pre-0163 & $4 / 13 / 2005$ & 2,660 & 3 & 18.08 & 0.666 & 8.03 & 52.0 & 0.0009 \\
\hline 132 & Pre-0164 & $4 / 12 / 2005$ & 3,020 & 3 & 19.46 & 0.680 & 2.70 & 13.0 & 0.0083 \\
\hline 133 & Pre-0166 & $3 / 8 / 2005$ & 2,840 & 4 & 20.12 & 0.684 & 0.06 & 163 & 0.0038 \\
\hline 134 & Pre-0171 & $4 / 12 / 2005$ & 1,640 & 3 & 19.42 & 0.655 & 0.00 & 27.7 & 0.2865 \\
\hline 135 & Ral-0196 & $3 / 25 / 2005$ & 2,680 & 3 & 17.77 & 0.652 & 1.32 & 130.1 & 0.0000 \\
\hline 136 & Ral-0220 & $5 / 13 / 1997$ & 2,850 & 1 & 17.88 & 0.652 & 0.10 & 34.1 & 0.0000 \\
\hline 137 & Ral-0221 & $5 / 27 / 1998$ & 2,584 & 2 & 15.93 & 0.576 & 0.07 & 775 & 0.0000 \\
\hline 138 & Ran-0260 & $3 / 10 / 2005$ & 4,400 & 3 & 19.19 & 0.657 & 0.26 & 3.7 & 1.2004 \\
\hline 139 & Ran-0275 & $3 / 7 / 2005$ & 2,120 & 2 & 19.06 & 0.669 & 0.06 & 18.3 & 0.1470 \\
\hline 140 & Ran-0276 & $6 / 19 / 2003$ & 3,130 & 1 & 5.63 & 0.242 & 0.00 & 0.4 & 44.9857 \\
\hline 141 & Ran-0278 & $7 / 9 / 2003$ & 2,800 & 3 & 18.22 & 0.639 & 0.16 & 0.6 & 8.5474 \\
\hline 142 & Roa-0093 & $4 / 11 / 2005$ & 1,000 & 3 & 23.63 & 0.765 & 0.00 & 0.4 & 0.2310 \\
\hline 143 & Sum-0099 & $5 / 22 / 1997$ & 1,956 & 1 & 19.14 & 0.683 & 0.22 & 28.9 & 0.0004 \\
\hline 144 & Sum-0103 & $6 / 18 / 1997$ & 2,658 & 1 & 20.13 & 0.685 & 0.09 & 4.7 & 0.0024 \\
\hline 145 & Sum-0105 & $6 / 18 / 1998$ & 2,380 & 1 & 32.88 & 0.918 & 2.54 & 41.1 & 0.0021 \\
\hline 146 & Tay-0127 & $4 / 12 / 2005$ & 1,320 & 3 & 24.24 & 0.793 & 0.00 & 25.6 & 0.0473 \\
\hline 147 & Tuc-0124 & $3 / 9 / 2005$ & 4,260 & 3 & 20.28 & 0.705 & 5.69 & 20.3 & 0.0000 \\
\hline 148 & Tuc-0125 & $3 / 9 / 2005$ & 3,280 & 4 & 18.66 & 0.670 & 3.01 & 4.0 & 0.0000 \\
\hline 149 & Tyl-0092 & $5 / 5 / 2004$ & 1,020 & 3 & 30.09 & 0.948 & 8.58 & 9.4 & 0.0000 \\
\hline 150 & Tyl-0093 & $5 / 6 / 2004$ & 850 & 4 & 20.34 & 0.698 & 0.15 & 993 & 0.0002 \\
\hline 151 & Ups-0181 & $3 / 7 / 2005$ & 2,060 & 4 & 19.90 & 0.691 & 0.13 & 54.2 & 0.0010 \\
\hline
\end{tabular}


Table 1. Summary of averaged dissolved-gas compositions (nitrogen, argon, oxygen, carbon dioxide, and methane) in water samples from wells and springs in West Virginia, 1997-2005.-Continued

[Map no., general reference number for map; rech. elev., recharge elevation is land surface; ft, feet; $\mathrm{n}_{\mathrm{dg}}$, number of dissolved-gas samples averaged; $\mathrm{N}_{2}$, nitrogen; Ar, argon; $\mathrm{O}_{2}$, oxygen; $\mathrm{CO}_{2}$, carbon dioxide; $\mathrm{CH}_{4}$, methane; $\mathrm{mg} / \mathrm{L}$, milligrams per liter; ${ }^{*}$, spring site]

\begin{tabular}{|c|c|c|c|c|c|c|c|c|c|}
\hline $\begin{array}{c}\text { Map } \\
\text { no. }\end{array}$ & Station name & Date & $\begin{array}{l}\text { Rech. elev. } \\
\text { (ft) }\end{array}$ & $\mathbf{n}_{\mathrm{dg}}$ & $\mathrm{N}_{2}(\mathrm{mg} / \mathrm{L})$ & $\operatorname{Ar}(\mathrm{mg} / \mathrm{L})$ & $\mathrm{O}_{2}(\mathrm{mg} / \mathrm{L})$ & $\mathrm{CO}_{2}(\mathrm{mg} / \mathrm{L})$ & $\mathrm{CH}_{4}(\mathrm{mg} / \mathrm{L})$ \\
\hline 152 & Way-0140 & $3 / 22 / 2005$ & 1,100 & 3 & 19.76 & 0.683 & 0.00 & 12.6 & 14.4221 \\
\hline 153 & Way-0143 & $3 / 22 / 2005$ & 1,000 & 3 & 22.43 & 0.786 & 0.00 & 9.3 & 9.7212 \\
\hline 154 & Way-0146 & $3 / 22 / 2005$ & 1,020 & 3 & 17.63 & 0.650 & 0.00 & 3.7 & 21.9988 \\
\hline 155 & Wet-0111 & 5/3/1999 & 1,220 & 1 & 22.24 & 0.729 & 0.07 & 111.3 & 0.0012 \\
\hline 156 & Wet-0133 & $4 / 13 / 2005$ & 1,080 & 3 & 15.30 & 0.593 & 0.00 & 0.2 & 7.9887 \\
\hline 157 & Woo-0185 & 5/6/1999 & 700 & 1 & 22.26 & 0.759 & 0.07 & 25.6 & 0.0009 \\
\hline 158 & Woo-0196 & 4/29/1999 & 780 & 1 & 20.03 & 0.687 & 0.06 & 33.2 & 0.0014 \\
\hline 159 & Woo-0213 & $3 / 15 / 2005$ & 700 & 4 & 18.74 & 0.649 & 1.23 & 23.3 & 0.0226 \\
\hline 160 & Wyo-0060 & 6/6/2001 & 2,240 & 2 & 15.16 & 0.524 & 0.00 & 3.5 & 23.5714 \\
\hline 161 & Wyo-0062 & 6/6/2001 & 2,120 & 4 & 53.98 & 1.124 & 0.14 & 40.1 & 18.2007 \\
\hline 162 & Wyo-0263 & 6/5/2001 & 1,880 & 4 & 13.82 & 0.449 & 0.00 & 16.5 & 34.6053 \\
\hline 163 & Wyo-0265 & $6 / 7 / 2001$ & 1,800 & 3 & 19.70 & 0.645 & 0.01 & 47.9 & 7.0480 \\
\hline 164 & Wyo-0268 & $6 / 4 / 2001$ & 1,560 & 1 & 8.37 & 0.322 & 0.00 & 12.8 & 57.4555 \\
\hline 165 & Wyo-0269 & 6/5/2001 & 1,720 & 3 & 13.97 & 0.496 & 0.00 & 15.9 & 35.3647 \\
\hline 166 & Wyo-0270 & 6/7/2001 & 2,520 & 4 & 18.58 & 0.669 & 0.03 & 44.0 & 3.4398 \\
\hline 167 & Wyo-0271 & $6 / 22 / 2005$ & 1,170 & 4 & 18.00 & 0.645 & 0.00 & 29.5 & 11.8826 \\
\hline 168 & Wyo-0273 & $7 / 18 / 2005$ & 2,360 & 4 & 19.46 & 0.679 & 0.08 & 27.4 & 0.0000 \\
\hline 169 & Wyo-0274 & 8/2/2005 & 2,050 & 4 & 17.87 & 0.674 & 0.09 & 23.5 & 6.1080 \\
\hline 170 & Wyо-0275 & $8 / 2 / 2005$ & 1,800 & 4 & 19.33 & 0.679 & 0.00 & 32.6 & 0.5580 \\
\hline 171 & Wyo-0276 & $8 / 3 / 2005$ & 2,500 & 4 & 18.37 & 0.630 & 0.15 & 38.6 & 2.6887 \\
\hline
\end{tabular}




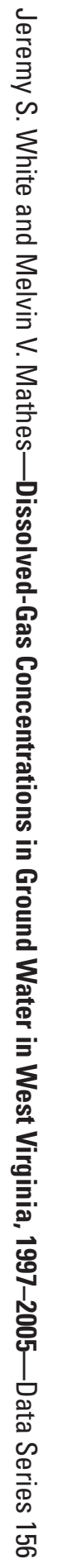

